\title{
Optical Conductivity and Dielectric Response of an Organic Aminopyridine NLO Single Crystal
}

\author{
T. Arumanayagam, P. Murugakoothan* \\ PG and Research Department of Physics, Pachiyappa’s College, Chennai, India. \\ *Corresponding author: murugakoothan03@yahoo.co.in
}

\begin{abstract}
This paper explores the correlation of electro-optical properties with dielectric properties of an organic single crystal. The optical constants of the organic aminopyridine single crystal have been studied. The second harmonic generation efficiency of the grown crystal, based on powder measurement, is 2.9 times higher than that of KDP. The real and imaginary part of the complex refractive index and dielectric constant of the crystal were determined. The optical and electrical conductivity of the grown crystal were studied.
\end{abstract}

Keywords: Nonlinear optical materials, Optical conductivity, Dielectric Properties,

\section{INTRODUCTION}

In recent years, researchers devoted much attention to nonlinear photonic crystals as their use in photonic band gap materials for controlling and molding the flow of light. The growth of research in nonlinear optics (NLO) is closely linked to the rapid technological advances that have occurred in related fields such as ultra-fast phenomena, optical communication and optical storage devices [1]. Organic nonlinear optical crystals which possess a good second harmonic generation efficiency due to their high optical band gap and low dielectric constant are in rich demand in optical storage devices, colour display units and optical communication systems etc [2]. It has been already reported that the pyridinium acceptor shows large second harmonic nonlinearity [3]. Recently optical properties of aminopyridine complexes and its suitability for the optoelectronic devices fabrications were reported $[4,5]$. In the present work, we have made an analysis of electro-optical properties correlated with dielectric properties of 2-aminopyridine 4-aminobenzoate single crystal.

\section{EXPERIMENTS}

The high optical quality 2-aminopyridine 4-aminobenzoate (APAB) single crystal was grown by slow evaporation technique. The compound was prepared by the reaction of 
2-aminopyridine (20 milli mol) and 4-aminobenzoic acid (10 milli mol) in (1:1) water methanol. The solution was heated with reflux about the temperature of $50{ }^{\circ} \mathrm{C}$ for 3 hours. A good optical quality single crystal of APAB was harvested after a period of 20 days and is shown in Figure 1(a). The unit cell dimension of APAB crystal was identified by single crystal X-ray diffraction analysis using Enraf Nonius FR 590 diffractometer with Mo Ka $(\lambda=0.7170 \AA)$ radiation. The cut and polished $2 \mathrm{~mm}$ thickness crystal was subjected for transmittance studies by using Model 1601 spectro-photometer. The second harmonic generation (SHG) efficiency of APAB was measured by Kurtz and Perry powder technique. The same thickness samples were used for the analysis of dielectric measurements for various frequencies and temperature using a HIOKI 3532-50 LCR HITESTER.

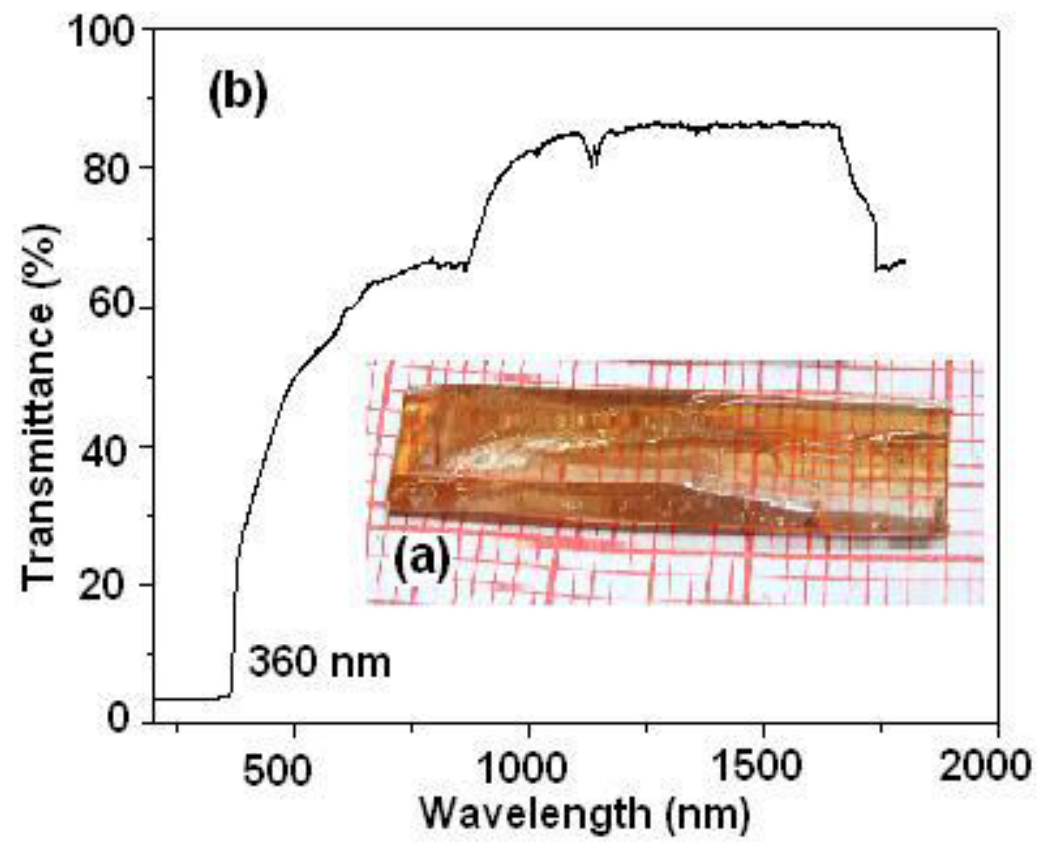

Fig. 1(a). Photograph of as grown single crystal. (b). UV-Vis-NIR transmittance spectra of APAB crystal.

\section{RESULT AND DISCUSSION}

The grown crystals were subjected to single crystal X-ray diffraction analysis. The crystal belongs to the triclinic crystallographic system. The obtained lattice parameter values are in good agreement with the reported literature values [6].

\subsection{Linear and Nonlinear Optical Studies}

Optical transmittance range and transparency cut off wavelength of the crystal are important factors for optical applications. Figure 1(b) which shows that there is no transmission up to $367 \mathrm{~nm}$ and it reveals that the crystal absorbs the entire UV region and transmit the visible and NIR region. The optical band gap energy of the grown crystal was calculated using the 
formula $E_{g}=1240 / \lambda(\mathrm{nm})$ in $\mathrm{eV}$, where $\lambda$ is the lower cut off wavelength $(367 \mathrm{~nm})$. The band gap of the APAB crystal is found to be $3.37 \mathrm{eV}$. As this crystal exhibits wide transmission range, starting from $367 \mathrm{~nm}$ onwards, it can be used for optical applications including the second harmonic generation of $\mathrm{Nd}$ : YAG laser of fundamental wavelength $\lambda=1064 \mathrm{~nm}$. It is also interesting to note from the spectrum that this crystal absorbs UV radiation and hence APAB crystal can be used as an effective UV shelter [7]. The measured transmittance $(\mathrm{T})$ data was used to calculate the absorption coefficient $(\alpha)$ from the following relation,

$$
\alpha=\frac{2.303 \log \left(\frac{1}{\pi}\right)}{t}
$$

where $t$ is the thickness of the crystal. The dependence of absorption coefficient on photon energy was analyzed in the absorption regions to obtain the detailed information about the energy band gap of the crystal. Figure 2(a) shows the variation of absorption coefficient $(\alpha)$ as a function of photon energy at room temperature. From the graph it is evident that the absorption coefficient varies from 6.5-16.4 $\mathrm{cm}^{-1}$ with increasing photon energy of 3.2-3.5 eV. The optical constants such as the refractive index (n) and the extinction coefficient (k) have also been estimated using the formula as reported earlier [8]. Figure 2(b) shows the plot of refractive index as a function of wavelength and it could be noticed that the refractive index decreases abruptly as the wavelength increases and gets saturated beyond the wavelength of $450 \mathrm{~nm}$. The refractive index of grown APAB crystal for longer wavelength (visible region) was calculated to be 2.36. From the calculated values of $\mathrm{n}$ and $\mathrm{k}$, the real and imaginary functions of dielectrics were also determined. The complex dielectric constant $\left(\varepsilon=\varepsilon_{\mathrm{r}}+\varepsilon_{\mathrm{i}}\right)$ characterizes the optical properties of the crystals and is calculated using the expressions as reported earlier [9]. The real and imaginary parts of the dielectric constant of the grown crystal were determined and shown in Figure 3(a). From the graph it is clear that both real and imaginary part of dielectric constant increases with increase of photon energy. The real part of the dielectric constant increases linearly with higher value than the imaginary part. The lower value of dielectric constant with wide band gap of APAB crystal suggests the suitability of optoelectronic devices.

The optical conductivity is one of the powerful tools for studying the electronic states in materials. The frequency dependence of dielectric reflects the fact that a material's polarization does not respond instantaneously to an applied field. For this reason, dielectric constant is often treated as complex function of the frequency of the applied field. A perfect dielectric is a material that has no conductivity. However the grown crystals associated with low dielectric loss inhibit the propagation of electromagnetic energy which aided conductivity. According to the one component or anomalous Drude model, both the carrier relaxation time and its effective mass of the charges are assumed to be as the functions of photon frequency $(\omega)$. But for alternative, multi component model, the real part of the optical conductivity $(\sigma)$ of the crystal was calculated using the following relations $[10,11]$ :

$$
\sigma=\frac{\omega}{4 \pi} \operatorname{Im}(\varepsilon)
$$


where the value of $\operatorname{Im}(\varepsilon)$ is given by,

$$
\operatorname{Im}(\varepsilon)=\frac{c^{2}}{\omega^{2}\left(\mu_{Y}\right)}(k \alpha) \text {. }
$$

where $\mu_{\mathrm{r}}$ is the relative permeability. For most crystalline materials $\mu_{\mathrm{r}}$ is very close to 1 at optical frequencies. On substuting the value of $\operatorname{Im}(\varepsilon)$ in Eq. (2)

$$
\sigma=\frac{a n c}{4 \pi}
$$

where c is the velocity of light. The plot between the optical conductivity against photon energy (hv) was depicted in Figure 3(b). The spectrum indicates that the optical conductance increases with the increase of photon energy.

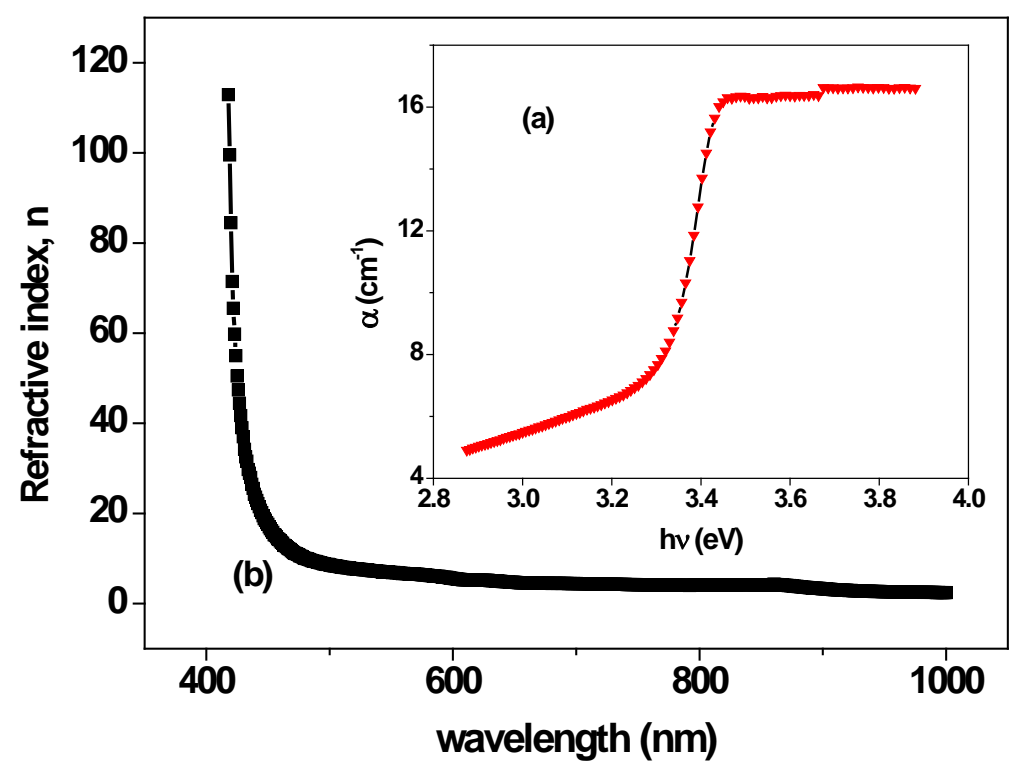

Fig. 2(a). Plot of absorption coefficient against photon energy. (b). Plot of refractive index against wavelength for APAB crystal.

For the second harmonic generation efficiency, a fundamental beam of wavelength $1064 \mathrm{~nm}$ with a pulse duration of $10 \mathrm{~ns}$ and frequency repetition of $10 \mathrm{~Hz}$ from Q-switched Nd:YAG laser was used as the source and passed through the powder sample [12]. The SHG behavior was confirmed from the output of the laser beam which had bright green emission $(\lambda=532 \mathrm{~nm})$ from the powder sample. The second harmonic signal of $32 \mathrm{mV}$ was obtained for an input energy of $31 \mathrm{~mJ} /$ pulse, while the standard KDP crystal gives a SHG signal of 11 $\mathrm{mV}$ for the same input energy. It shows that the SHG efficiency of APAB is 2.9 times that of standard NLO material, KDP. 


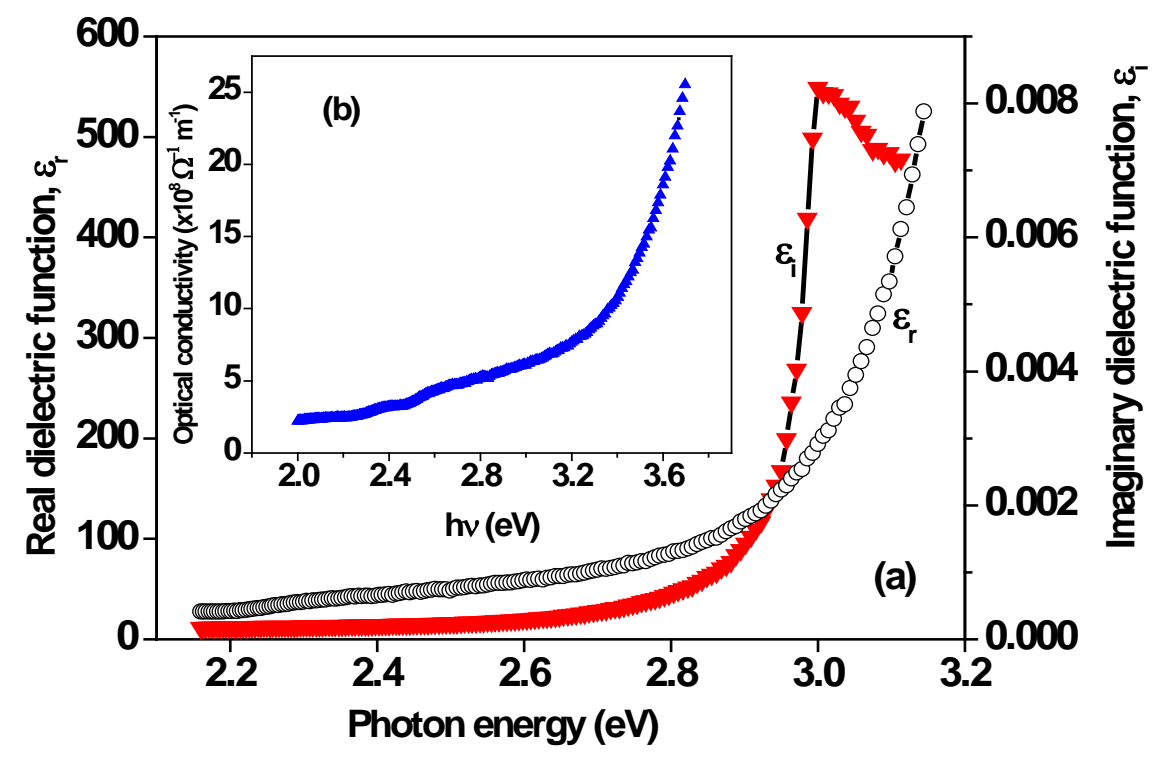

Fig. 3(a). Plot of $\varepsilon_{\mathrm{r}}$ and $\varepsilon_{\mathrm{i}}$ as a function of photon energy of APAB crystal. 3(b). Optical conductivity against photon energy of APAB crystal

\subsection{Dielectric Studies}

The dielectric properties are correlated with electro-optic properties of the crystals [13]. The dielectric constant $\left(\varepsilon_{\mathrm{r}}\right)$ was calculated by using the relation, $\varepsilon_{\mathrm{r}}=\mathrm{Ct} / \mathrm{A} \varepsilon_{0}$, where $\varepsilon_{0}$ is the permittivity of the free space, $\mathrm{C}$ is the capacitance and $\mathrm{A}$ is the area of cross section of the sample. Figure 4(a) and 4(b) shows the plot between the calculated dielectric constant and dielectric loss with respect to frequency for APAB crystal, respectively. The graph reveals that the dielectric constant has higher value at lower frequencies and almost constant at higher frequencies (beyond $100 \mathrm{kHz}$ ), calculated to be 32.5. The magnitude of dielectric constant depends on degree of polarization and the charge displacement in crystal. The decrease in dielectric constants at higher frequencies is attributed to the absence of space charge polarization near the grain boundary interface [14, 15]. For a material to be a potential candidate for NLO applications, dielectric loss ( $\tan \delta$ ) must also be kept as low as possible. From the graph, it is clear that the APAB crystal exhibit very low dielectric loss at high frequencies and can be used for NLO applications effectively [16].

The crystal was subjected to an external electric field. Then, generally, a redistribution of charges occurs and currents are induced. The ac conductivity of the samples was calculated using the formula [17];

$$
\begin{array}{ll}
\sigma_{\mathrm{ac}} & = \\
\varepsilon_{0} \varepsilon_{\mathrm{r}} \omega \tan \delta
\end{array}
$$

where $\omega$ be the angular frequency $(\omega=2 \pi v)$. Figure 5 , shows the variations of ac conductivity of APAB crystal with various frequencies. The conductivity is almost zero up 
to $10 \mathrm{kHz}$ and then increases with the increase of frequency. The low value of electrical conductivity is the effect of decrease in mobility of the charge carriers due to ionic size, which leads to the change in electronic band structure. At higher frequency the ac conductivity increases sharply. It reveals that the electrical conductivity is proportional to mobility and carrier concentrations through the well known relation $\sigma=\mathrm{n}_{\mathrm{d}} \mathrm{e} \mu_{\mathrm{e}}$, where $\mu_{\mathrm{e}}$ is the mobility of electron and $n_{d}$ is the number density of electron. Thus the optical conductivity of the grown APAB crystal increases by increased with increase in applied energy.

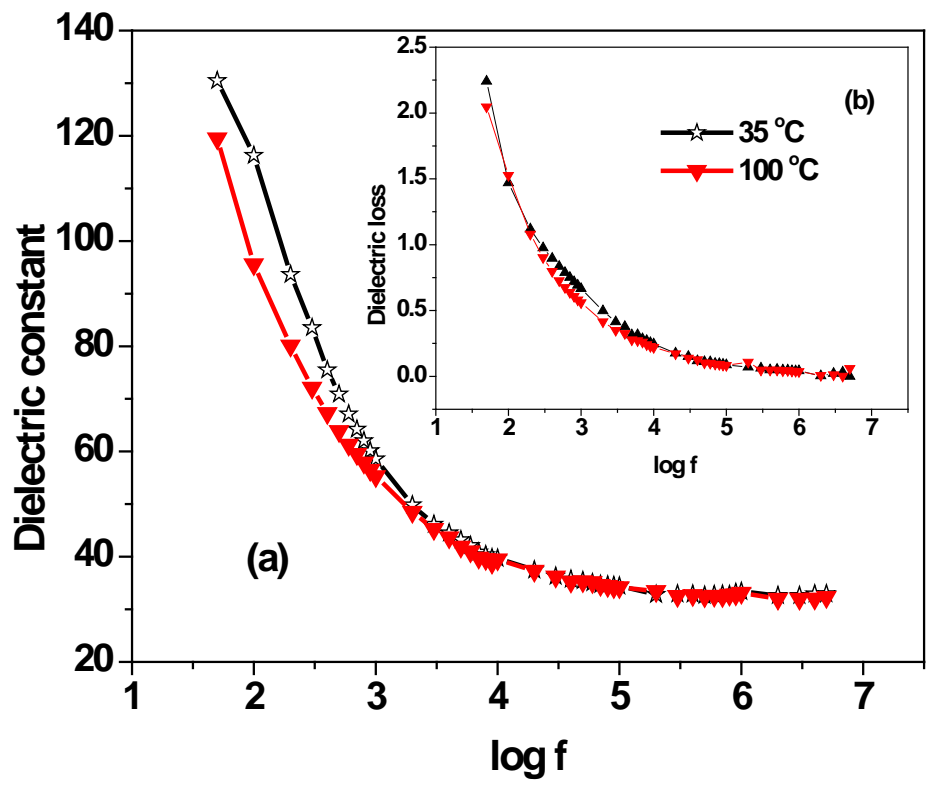

Fig. 4(a). Variation of dielectric constant with frequency.

(b). Variation of dielectric loss with frequency.

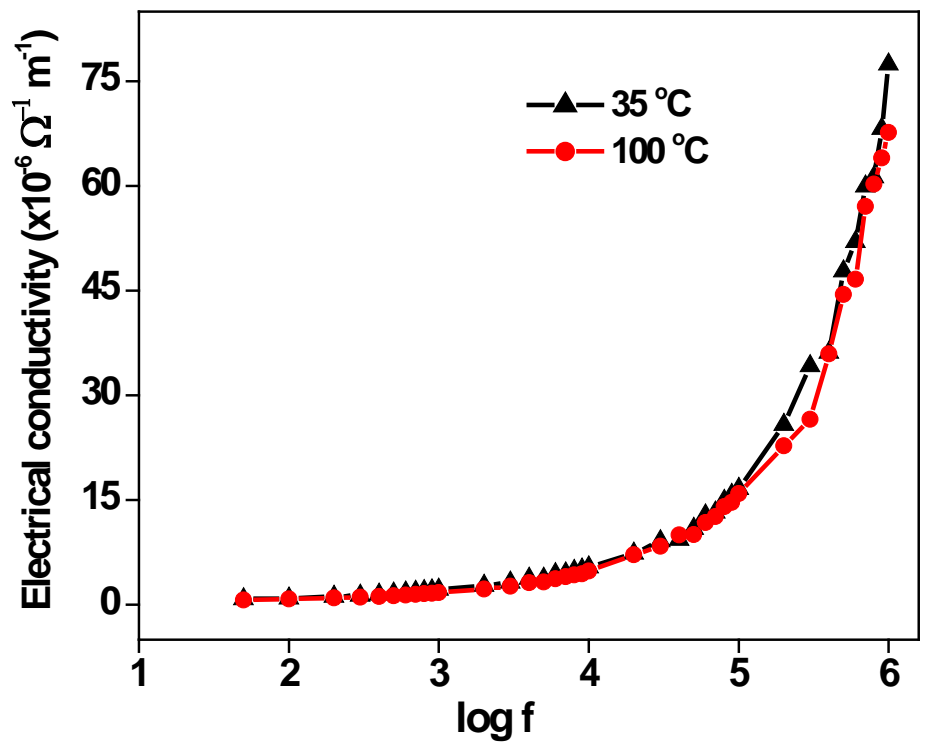


Fig. 5. Plot of electrical conductivity versus frequency of APAB crystal. 


\section{CONCLUSION}

Under the reaction of 2-aminopyridine and 4-aminobenzoic acid with the ratio 2:1, high quality nonlinear optical crystal was grown using slow evaporation technique. The optical transmittance, band gap and optical constants of the grown crystal were studied. The complex dielectric functions of the APAB crystal were studied. The SHG efficiency of the grown APAB crystal is 2.9 times that of KDP. The optical and electrical conductivity of the sample was studied and it reveals that the conductivity increases with increase of photon energy. The dielectric constant of the grown crystal is calculated to be 32.5 at higher frequency. The high optical transmittance and band gap with low dielectric constant and low dielectric loss suggest that the APAB crystal could be used in nonlinear optoelectronic devices.

\section{REFERENCES}

[1] Yari S. Kivshar, Optics Express, 16, 22126-22128 (2008).

[2] D.S. Chemla, J. Zyss, Nonlinear Optical properties of Organic materials and Crystals, Academic press, New York, 1987.

[3] Sabir H. Mashraqui, Rajesh S. Kenny, Shailesh G. Ghadigaonkar, Anukrishnan, Mily Bhattacharya, Puspendu K. Das, Optical Materials, 27, 257-260 (2004).

[4] Bhuvana K. Periyasamy, Robinson S. Jebas, Balasubramanian Thailampillai, Materials Letters, 61 (2007) 1489-1491.

[5] K. P. Bhuvana, S. Robinson and T. Balasubramanian, Cryst. Res. Technol., 45, 299-302 (2010).

[6] A.B. Jonna, M.J. Zaworotko, Crystal Growth and Design, 5, 1169-1179 (2005).

[7] Science News Online (6/6/98): Melanoma Madness The scientific flap over sunscreens and skin cancer -- Chemical studies (accessed 10/1/2009, 2009).

[8] K. Goksen, N.M. Gasanly and H. Ozkan, Acta Physica Polonica, 112, 93-100 (2007).

[9] Fahrettin Yakuphanoglu, Hilmi Erten, Optica applicata, 35, 969-976 (2005).

[10] A. Lucarelli, S. Lupi, P. Calvani and P. Maselli, Physical Review B, 65, 054511,1-7 (2002).

[11] E.I. Ugwu, A.S. Olayinka and F.I. Olabode, J. Eng. Applied Sci., 4, 126-131 (2009).

[12] S. K. Kurtz and T. T. Perry, J. Appl. Phys. 39, 3798 (1968).

[13] P. Mythili, T. Kanagasekaran, S. Stella Mary, D. Kanjilal and R. Gopalakrishnan, Nuclear Instruments and Methods in Physics Research Section B, 266, 1737-1740 (2008).

[14] K.V. Rao, A. Smakula, J. Appl. Phys. 36, 2031-2038 (1965).

[15] B. Narasimha, R.N. Choudhary, K.V. Roa, Mater.Sci. 231416 (1988).

[16] D. Balasubramanian, P. Murugakoothan, R. Jayavel, J. Cryst. Growth,312, 1855-1859 (2010).

[17] M. Vimalan, A. Ramanand and P. Sagayaraj, Cryst. Res. Technol, 42, 1091-1096 (2007). 